\title{
SpARF4 reduces cadmium accumulation by negatively regulating SpABCG14 and SpACO4 in the cadmium/zinc hyperaccumulator Sedum plumbizincicola
}

\author{
Dong $\mathrm{Xu}^{1}$, Zhuchou $\mathrm{Lu}^{1}$, Longhua $\mathrm{Wu}^{2}$, Guirong Qiao ${ }^{3}$, Wenmin Qiu ${ }^{3}$, Han Xiaojiao ${ }^{4}$, and \\ Renying zhuo ${ }^{5}$ \\ ${ }^{1}$ Research Institute of Subtropical Forestry Chinese Academy of Forestry \\ ${ }^{2}$ Institute of Soil Science, Chinese Academy of Sciences \\ ${ }^{3}$ State Key Laboratory of Tree Genetics and Breeding, Chinese Academy of Forestry \\ ${ }^{4}$ State Key Laboratory of Tree Genetics and Breeding, Chinese Academy of Forestry, \\ Xiangshan Road, Beijing, 100091, China \\ ${ }^{5}$ The Research Institute of Subtropical Forestry, Chinese Academy of Forestry
}

March 12, 2021

\begin{abstract}
Root development and apoplastic transport are respectively important for cadmium (Cd) absorption and transportation, which profoundly influence $\mathrm{Cd}$ bioremediation. However, molecular mechanisms underlying the two processes are not completely understood. In this study, we demonstrated that auxin response factor 4 (SpARF4) from a Cd hyperaccumulator Sedum plumbizincicola was a negative regulator for these processes. SpARF4 positively regulated by auxin was highly expressed in xylem. Overexpression of SpARF4 significantly decreased vessel area and declined lignin content of S. plumbizincicola. Meanwhile, less adventitious roots were found, and lateral root development was delayed in transgenic plants. Furthermore, ethylene production and auxin transportation were impaired. More importantly, SpARF4 negatively regulated Cd content of xylem saps and aerial tissues. Combining dual-LUC reporter, Y1H and qRT-PCR assays, SpARF4 was a repressor for two downstream genes (SpABCG14 and SpACO4) which influenced vascular bundle development and ethylene production, respectively. PIN1, 2, 3, 7 were downregulated and slowed down local auxin accumulation rate, which suspended root development. These results indicate that SpARF4 can decelerate Cd transportation rate from roots to aerial parts and reduce Cd content of aboveground tissues by delaying the root development and decreasing vessel area.
\end{abstract}

\section{Introduction}

Two different attitudes are adopted by people toward cadmium (Cd) accumulation in plants. On one hand, Cd accumulation should be decreased in crops (especially the edible portions) for ensuring food security (Sasaki et al., 2012; Rizwan et al., 2016; Lu et al., 2019). On the other hand, plants used for bioremediation should accumulate more $\mathrm{Cd}$ in their aerial parts for removing Cd contamination (Ali et al., 2013; Evangelou et al., 2015; Luo et al., 2016). Cd absorption and transportation of plants are involved in both cases. Therefore, understanding the molecular mechanism underlying $\mathrm{Cd}$ absorption and transportation is crucial for ensuring food security and environmental restoration.

Root morphology can response to Cd stress (Daud et al., 2009), which may lead to a change in Cd absorption ( $\mathrm{Li}$ et al., 2009). For example, in hyperaccumulating-ecotypesSedum alfredii (HE, a famous Cd hyperaccumulator), Yang et al. (2004) pointed out that although the maximum root length was significantly reduced when Cd levels $>50 \mu \mathrm{mol} \cdot \mathrm{L}^{-1}$, more LRs were grown (Yang et al., 2004). Most importantly, Cd 
absorption was simultaneously increased. In line with this idea, it is also reported that apoplastic pathway via LRs contributed to Cd accumulation (Tao et al., 2016). In this study, our attention is focused on root development, especially lateral roots (LRs) and adventitious roots (ARs). Because LRs are mainly relevant with Cd absorption of plants (Redjala et al., 2011; Tao et al., 2016; Xie et al., 2019), while ARs are related with LR numbers.

Auxin plays crucial roles in initiation of ARs and LRs, especially its transportation and local accumulation (Casimiro et al., 2001; Vidoz et al., 2010; Sukumar et al., 2013; Du and Scheres, 2017). Auxin response factors (ARFs ), as important components for auxin pathway, are thus significant for root development (Okushima et al., 2007; Lee et al., 2019). To date, largely based on studies of Arabidopsis, ARF2, 3, 4, 5, 7, $6,8,17$ and 19 have been proved to be associated with root development (Wilmoth et al., 2005; Gutierrez et al., 2009; De Smet et al., 2010; Marin et al., 2010; Xi et al., 2018). In addition, the root initiation seems to be depended on expression levels of ARFs. For example, miR167 downregulates GmARF8 to increase LRs (Wang et al., 2015). Overexpression of AtARF17 decreased the amount of ARs (Sorin et al., 2005). A regulatory module (containing $A R F 4$ ) has dramatic impacts on LRs development and remains highly conservative between different species (Marin et al., 2010; Hobecker et al., 2017; He et al., 2018). ARF4 is a repressor for LRs in normal condition (Marin et al., 2010). But,ARF4 was downregulated and released LRs restriction in adverse situation (He et al., 2018). However, as a typical transcription factor, how ARF 4 regulates its downstream genes to control LRs development requires further investigation.

Notably, most of auxin which induces LRs initiation is originated from young shoot organs (Aloni et al., 2006), suggesting the importance of polar auxin transportation for LRs development. Intercellular transport of auxin is mainly mediated byPIN, $A B C B$ and $A U X / L A X$ families (Geisler et al., 2005; Paponov et al., 2005; Péret et al., 2012). Over the past decades, numerous studies were focused on PIN genes, including their evolution, expression locations and functions (Blilou et al., 2005; Paponov et al., 2005). Up to date, eight PIN genes are identified in Arabidopsis (Paponov et al., 2005). Among them, PIN1, 2, 3, 4, 7 control root patterns and their mutants exhibited developmental retardation or even deficiency of LRs (Benková et al., 2003), suggesting PINs are necessary for LRs. Moreover, expression levels of them could be regulated by ethylene (Lewis et al., 2011). Therefore, adverse environments (such as Cd stress) which induce ethylene promote more LRs emergence, indicating that LRs development seems to be an important strategy for stress adaptation of plants (Yang et al., 2004; He et al., 2005). However, in turn, excessive ethylene is produced with the increase of stress time, which blocks LRs initiation (Xiong et al., 2006; Negi et al., 2008). Normal LRs development are processing in the presence of ethylene within a certain range. In other words, the factor which represses ethylene production under abiotic stress should be crucial for LRs growth. Thus, it is necessary to uncover the mechanism inhibiting ethylene production. Meanwhile, different factors (ARFs , PINs and ethylene) can all inhibit LRs, what is the relationships between them ?

After $\mathrm{Cd}$ accumulated in roots, timely transportation of $\mathrm{Cd}$ to aerial tissues is an important strategy for protecting roots. More efficient Cd transportation is a most striking difference betweenSedum plumbizincicola (previously known as HE) and S. alfredii (known as NHE, non-hyperaccumulator of Cd) (Lu et al., 2008), even though the two species have very closely genetic relationships (Jin et al., 2008). Up to data, apoplastic and symplastic pathway are responsible for Cd transportation (Luo et al., 2016). Most studies are concentrated on symplastic pathway (Park et al., 2012; Liu et al., 2017). However, recent studies demonstrated that the apoplastic pathway not only exerts their roles in Cd transportation (Tao et al., 2019) but also in Cd sequestration (ZHANG et al., 2010). Nevertheless, the molecular mechanism underlying this pathway has been elusive.

ABCG14in Arabidopsis is a crucial gene to profoundly influence the apoplastic pathway, because it is related with vessel cell development and lignin biosynthesis (Ko et al., 2014; Zhang et al., 2014; Zhao, 2016). On one hand, vessel cells are relevant with inorganic ion transportation. On the other hand, although the functions of lignin in vivo in binding $\mathrm{Cd}$ were not clear, it exhibits extraordinary abilities for Cd absorption in vitro experiment (Demirbas, 2004; Harmita et al., 2009). Moreover, lignin biosynthesis genes can response to Cd stress (van de Mortel et al., 2008). Thus, ABCG14 has a potential ability to affect Cd bioremediation, 
however, regulatory mechanism about it remains largely unknown. In this study, S. plumbizincicola and $S$. alfrediiwere chosen as materials to identify gene functions. SpABCG14 whose protein domain remained highly conserved with $A B C G 14$ was isolated from S. plumbizincicola (Fig. S1) and many binding sites associated with various transcription factors were found in its promoter (Fig. S2), such as ARFs binding element, AuxRE. It revealed that $S p A B C G 14$ may be controlled by these transcription factors. Among them, $A R F s$ are selected to understand whether and how $A R F$ members could modify $S p A B C G 14$ expression to influence apoplast pathway.

Therefore, our main aims in this study are i) to choose the candidate gene from $A R F$ family by bioinformatics analysis and to verify the relationship between it and $S p A B C G 14$; ii) to analyze its functions in root development; iii) to identify its influence on $\mathrm{Cd}$ transportation and accumulation.

\section{Materials and methods}

\section{Plant materials and growth conditions}

Materials of S. plumbizincicola and S. alfredii were collected as previously described (Liu et al., 2017). Some of them were treated for tissue culture. Shoots of other S. plumbizincicolawere cut and used in the hydroponic treatment for two weeks. The rooted clones were transplanted in nutrient soils and cultured for 6 months before Cd treatment with 16-hour light/8-hour dark cycles. After that, the suitable shoots were harvested and rooted in nutrient solutions containing Murashige and Skoog (MS) for two weeks. These rooted plants were treated with $400 \mu \mathrm{M} \mathrm{CdCl} 2$ for $0,0.5,1,2,4,8$ and $12 \mathrm{~h}$, respectively.

\section{Identification of $A R F$ genes using genome data}

Protein sequence data of $S$. plumbizincicola and other species (including Kalanchoe laxiflora, Kalanchoe fedtschenkoi ,Arabidopsis thaliana , Populus trichocarpa ,Selaginella moellendorffii, Zea mays , Oryza sativa ,Marchantia polymorpha, Ostreococcus lucimarinus ,Chromochloris zofingiensis ) were downloaded from Phytozome (https://phytozome.jgi.doe.gov/pz/portal.html\#!bulk?org=Org_Athaliana). ARF genes were screened from these protein data on Pfam platform using Auxin_resp domain (PF06507.12) and the sequences with E-values equal or more to 1E-5 were discarded. After that, all of genes in this study were named, according to the conservation between them and their homologous genes in Arabidopsis. Then, if two or more genes have the same homologous gene in Arabidopsis, they were named 1, 1.2 etc.

\section{Quantitative reverse transcription-PCR (qRT-PCR)}

Total RNAs were extracted from roots, stems and leaves of above Cd-treatment plants (three biological replicates per treatment) using an RNA extraction kit (RNAprep Pure Plant Kit, TIANGEN, Dalian, China). Next, first-strand cDNAs were produced using a cDNA synthesis kit (PrimeScript RT Master Mix, TAKARA, Beijing, China). These cDNAs were utilized for qRT-PCR using TB Green reagent (TB Green Premix Ex Taq, TAKARA, Dalian, China). The primers of $A R F$ genes were designed at the website PRIMER3 (http://primer3.ut.ee/). All primers including the reference gene (UBC9) were listed in Table 1S.

Analysis of Phylogenetic, positively selected sites, functional divergence and coexpression network

ARF protein sequences of S. plumbizincicola, K. laxifloraand K. fedtschenkoi were renamed after blasted in TAIR (https://www.arabidopsis.org/). Then, protein sequences alignment were made using the method of Mafft (http://mafft.cbrc.jp/alignment/software/) and Muscle (https://www.ebi.ac.uk/Tools/msa/muscle/). After that, phylogenetic tree was conducted by MEGA-X (Kumar et al., 2018) with the bootstrap 1000. Then the phylogenetic tree was applied in the software PAML (Yang, 2007) for positively selected sites calculation and the alignment file was produced by MEGA-X, with the method of clustal W (codons). Meanwhile, the software DAMBE was used for format conversion. M0, M3 and M7, M8 models were respectively applied for assuming variable selected pressure, according to the method of Liu et al. (2018) (Liu et al., 2018). Functional divergence analysis was made using the software of DIVERGE v3.0 (Gu, 2011). Coexpression network were conducted by Cytoscape software, based on the transcriptome data (Han et al., 2016). The 
corresponding genes related to hub genes were thus identified and then promoter cis-elements of these genes were further analyzed, combined with the data of transcriptome and genome, using TBtools and PlantCARE.

\section{Plasmid construction}

The open reading frame and $2 \mathrm{~kb}$ promoter of $S p A R F 4$ were amplified from cDNA and DNA of S. plumbizincicola, respectively. These products were cloned into pDONR222 and then were recombined into pK2GW7.0 and pMDC164 to generate 35S:SpARF4 andProSpARF4:GUS , respectively. Meanwhile, the full coding sequence of SpARF4 was assembled into the vectors of pGADT7-Rec (for Yeast one-hybrid assay, Y1H) and pGreenII 62-SK (for dual-LUC reporter system) using the sites of Sma I/BamH I andEcoR I/Hind III, respectively. Full-length promoter sequences (2kb) of SpABCG14 and $S p A C O 4$ were amplified from $S$. plumbizincicola and inserted into the vector of pGreenII 0800-LUC using Sal I/BamH I to formSpABCG14pro:LUC and $S p A C$ 4pro $_{\text {: }} L U C$. Meanwhile, to demonstrate the availability of our system, $35 \mathrm{~S}$ promoter was also cloned into pGreenII 0800-Luc to form 35S:LUC, as the efficiency of 35S promoter was stronger than that of plant promoters. All primers were listed in Table 1S.

\section{Plant transformation}

Explants for shoot induction in transgenic experiment were calluses ofS.plumbizincicola and leaves of S.alfredii. The constructs containing 35S:SpARF4 and ProSpARF4:GUS were introduced into EHA105 Agrobacterium tumefaciens strain by electroporation, respectively. Subsequently, the 35S:SpARF 4 was used for infecting S. plumbizincicola and S. alfredii. Meanwhile, the ProSpARF4:GUS was used for infecting S. plumbizincicola, and ProDR5:GUS vector was introduced into S. plumbizincicola. The A. tumefaciens -infected method was performed as described before (Liu et al., 2017), with minor modifications. Differentiation medium: MS $+2 \mathrm{mg} \cdot \mathrm{L}^{-1} 6$-benzylaminopurine $(6-\mathrm{BA})+0.3 \mathrm{mg} \cdot \mathrm{L}^{-1} 1$-naphthaleneacetic acid (NAA); rooting medium: $1 / 2 \mathrm{MS}+2 \mathrm{mg} \cdot \mathrm{L}^{-1} 3$-indolebutyric acid (IBA). The use of antibiotics is varied with different vectors. The concentrations of kanamycin and hygromycin were $30 \mathrm{mg} \cdot \mathrm{L}^{-1}$ and $20 \mathrm{mg} \cdot \mathrm{L}^{-1}$, respectively. After that, the well-rooted wild-type and transgenic plants were transplanted into soils and grown for further detection and experiments.

\section{Pot experiment and determination of Cd concentration in plants}

The overexpression lines (OE) of S. plumbizincicola with highest-expression level of SpARF4 (at least six lines) were cultured for $14 \mathrm{~d}$ using basic nutrient solution (Yang et al., 2004). Then, these plants were transplanted into plastic pots filled with $2 \mathrm{~kg}$ soil and watered every seven days (photoperiod 16h light/8h dark, day/night temperature $26 / 20 * \mathrm{C}$ ). The wild-type lines (WT) followed the same processes. After three months, these plants were cultured but without water for $14 \mathrm{~d}$. After that, these plants were supplied with $400 \mu \mathrm{mol} \cdot \mathrm{L}^{-1} \mathrm{Cd}$ solution for $48 \mathrm{~h}$. Aerial tissues of WT and $\mathrm{OE}$ were collected for measuring $\mathrm{Cd}$ concentration and three biological repeats. At the same time, removing the first drop, other xylem saps of these plants were collected in four hours using injectors and three biological repeats. Cd concentrations of these samples was determined as previously described (Liu et al., 2017).

\section{Measurement of IAA concentration and lignin content}

Plants of S.plumbizincicola were grown hydroponically for eight weeks. Then, these plants were divided into two groups which treated by 0 and $400 \mu \mathrm{mol} \cdot \mathrm{L}^{-1} \mathrm{CdCl}_{2}$ for seven days, respectively. The aboveground tissues of these plants were collected at the $0,1,4$ and $7 \mathrm{~d}$, respectively, for determination of lignin concentrations. Meanwhile, the samples which were cultured for seven days under Cd stress were prepared for IAA measurement. The determination IAA concentration was conducted as described preciously (Zhao et al., 2019). Lignin content was measured using the method of Redox titration (Pengcheng et al., 2008). In detail, the dried samples were soaked using $1 \%$ acetic acid solution and then the mixed solution containing ethanol and ether (volume ratio, 1:1). The sediments were evaporated to dryness in boiling water bath and treated by $72 \%$ sulfuric acid. Distilled water was used to clean the sediments. After that, $10 \%$ sulfuric acid and $0.1 \mathrm{~mol} \mathrm{~L}^{-1}$ potassium dichromate solution were added and the system was placed into boiling water bath. Then, adding $20 \% \mathrm{KI}$ and $0.5 \%$ starch solution to the mixture and using $0.2 \mathrm{~mol} \mathrm{~L}^{-1}$ sodium thiosulfate for 
titration.

\section{Y1H assay}

The coding region of $S p A R F 4$ was inserted into the vector of pGADT7-Rec (Clontech) to produce pGADT7SpARF4. A 20-bp fragment (as a unit) whose center was AuxRE motif (TGTCTC) was cloned from the promoters of $S p A B C G 14$ and $S p A C O_{4}$, respectively. Then four tandem copies of the two units were constructed and cloned into pHIS2 vectors to produce pHIS2-ABCG14 and pHIS2-ACO4, respectively (Liu et al., 2015). The two pairs (pGADT7-SpARF4/pHIS2-ABCG14 and pGADT7-SpARF4/pHIS2-ACO4) were cotransformed into AH109 (yeast strain) using LiAc-PEG3350 method. SD-Leu-Trp plates were used for transformants selection and SD-Leu-Trp-His plates supplemented with $30 \mu \mathrm{M} 3$-AT (3-amino-1,2,4,-triazole) were utilized for testing the interactions. Three biological repeats and three technical repeats for this process. The primers were contained in Table $1 \mathrm{~S}$.

\section{Transient transcriptional activities assay}

The transient transcriptional activity assays were performed as described previously (Liu et al., 2019), with minor modifications. The corresponding vectors were constructed as stated above. Lumazone imaging system (Mag Biosystems, USA) was used for image acquisition of luciferase in vivo. The images processing was accomplished by the software of ImageJ (https://imagej.net/Welcome). qRT-PCR were conducted for testing the expression level of $L U C$ and Ren. At least three biological repeats and technical repeats for each assay.

\section{Histochemical analyses}

$\beta$-glucuronidase (GUS ) staining was performed as described previously (Han et al., 2012). Phloroglucinol staining was conducted following the standard protocols (Zhang et al., 2012). All tissues sections were 30- $\mu$ m-thick, which observed and photographed using LEICA DM4000B/DFC450 and M165FC/DFC550. Vessel diameters and cross-section area of fifty vessel cells were measured by software of autoCAD (https://www.autodesk.com.cn/). After that, statistical analysis of these data was performed by SPSS 20.0.

\section{Auxin and ethylene inhibitors treatment}

The selection and application of NPA (naphthylphthalamic acid, auxin transport inhibitor), AVG (aminoethoxyvinylglycine, auxin synthesis inhibitor) and PZA (pyrazinamide, ethylene biosynthesis inhibitor) were referred to published studies (Scanlon, 2003; Soeno et al., 2010; Sun et al., 2017). In details, transgenic lines of ProSpARF4:GUS which were cultured under hydroponic conditions for 2 weeks were treated by $10 \mu \mathrm{M}$ NPA and $40 \mu \mathrm{M}$ AVG for $7 \mathrm{~d}$ and $3 \mathrm{~h}$, respectively. The seedings of $S$. alfredii were cultured in MS medium in the presence or absence of $100 \mu \mathrm{M}$ PZA for $14 \mathrm{~d}$. Three biologicals.

\section{Results}

\section{Lignin content was increased and IAA was decreased under Cd stress}

As $A B C G 14$ are related with lignin biosynthesis (Ko et al., 2014), while the functions of lignin in vivo about $\mathrm{Cd}$ accumulation were not so clear. lignin concentration in dry weight (DW) was measured during Cd stress. Meanwhile, Cd concentration in lignin was also tested. The results indicated that lignin concentration of DW under Cd stress was significantly higher than that without $\mathrm{Cd}$ treatment (Fig. 1a, $\mathrm{P}<0.01$ ). The concentration of lignin continued to be increased during Cd stress, even though, the increase did not reach a significant level (Fig. 1a, $\mathrm{P}<0.05$ ). Interestingly, there was an obviously difference in $\mathrm{Cd}$ concentration of lignin during $\mathrm{Cd}$ stress, indicating that slight changes in lignin can significantly enhance Cd content in DW (Fig. 1b, $\mathrm{P}<0.01$ ). Thus, genes related with lignin biosynthesis may trigger the variation in Cd content.

In order to clarify the roles of ARFs under Cd stress, we need to identify the influence of Cd on auxin, as ARFs functions were depended on auxin level ( $\mathrm{Li}$ et al., 2016). The results showed that IAA was seriously repressed in root (Fig. 1c, $\mathrm{P}<0.01$ ). similar trend was also found in stem (Fig. 1c, P <0.05). however, IAA content in leaf seemed not be affected by Cd treatment (Fig. 1c). 


\section{$S p A R F 4$ as a center in coexpression network underwent the positive selection}

Total 247 ARF genes in 11 species from four categories plants (phycophyta, moss, gymnosperm and angiosperm) were identified using pfam platform (PF06507.12), according to the published genome data (https://phytozome.jgi.doe.gov/pz/portal.html). The results revealed that ARF numbers were increased with plant evolution, and most of them were concentrated in terrestrial plants (Fig. 2A). More importantly, further analysis indicated that ARFsmay be involved in environmental stress responses. For example, promoter analysis showed that many elements related with abiotic stress (such as drought-inducibility element) were found in promoters of $23 S p A R F$ s in S. plumbizincicola (Fig. S3). In summary, we assumed that ARF gene family seemed to be relevant with the process of plant terrestrial adaptation and play crucial roles in protecting plants from abiotic stress. In order to verify this conclusion, we tested expression levels of $S p A R F s$ under Cd stimuli. The results revealed that expression patterns of SpARFswere changed during Cd stress, which indicated that $S p A R F s$ can response to Cd treatment (Fig. S4).

After analyzing ARFs in different species (Fig. 2A), we focused our attention on Crassulaceae family. ARFs in K. laxiflora , K. fedtschenkoi and S. plumbizincicola were used for constructing phylogenetic tree to analyze the functional divergence and adaptive selection (Fig. 2B). Only S. plumbizincicola in chosen three species was Cd hyperaccumulator. The results indicated that these ARFs were divided into four classes (Fig. 2B). Functional divergence analysis showed that the LRT values between the I class and other three classes reached a significant level (Table S1, P $<0.05$ ), indicating that Type I divergence sites may be distributed in the class I, during the evolution. Further evidence suggested that abundant positive selection sites were found in class I which containing SpARF3 and SpARF4 (Fig. 2B, Table S2). Furthermore, coexpression network containing five hub genes $(S p A R F 4,8,9,16.2$ and 19.2) was made using the transcriptome data under Cd stress (Fig. S5). The five genes were divided into two categories, according to the amount of shared related genes (Fig. S5A, B). Thus, from the point of our view, SpARF4 could be an ideal candidate gene, as it was a hub gene and underwent positive selection. Over 1000 genes in coexpression network were related with SpARF4 . Among of them, expression levels of 201 genes were changed during Cd stress (Correlation coefficient between gene expression and stress time $>0.9$ or $<-0.9$ ). In this study, our attention was mainly focused on three kinds of genes (transcription factors, transporters and phytohormone pathway). Finally, 11 genes including $S p A B C G 14$ and $\mathrm{SpACO}_{4}$ were identified (Fig. 2C, the red letters).

\section{$S p A R F 4$ positively regulated by auxin mainly expressed in vessel cells of xylem}

Expression sites of genes were closely associated with their functions. To investigate SpARF4expression patterns, $2 \mathrm{~kb}$ promoter sequences was assembled into a $G U S$ vector (pMDC164) (Fig. S6) and transformed intoS. plumbizincicola. The results revealed that GUS activities were detected in phloem and xylem (Fig. $3 \mathrm{~A}$, red arrows) and stronger signals were focused on vessel cells (Fig. 3B, the red arrow), which were similar to the expression locations of $A B C G 14$ (Ko et al., 2014), suggesting that there were possibilities for the relationship betweenSpARF4 and SpABCG14.

In general, genes in auxin pathway were controlled by auxin which was influenced by abiotic stress (Iglesias et al., 2010). Thus, in order to clarify the roles of SpARF4 in Cd stress, we need to identify the response of SpARF 4 to auxin. NPA and AVG were used to examine the relationship between SpARF 4 and auxin, as NPA can inhibit auxin transportation but not repress auxin biosynthesis while AVG can block auxin biosynthesis. We found that GUS signals were mainly focused on roots center in normal condition (Fig. 3C-1; Fig. S7A, B). Moreover, the signals were not only distributed in root tips but also in other parts (Fig. 3C-1, the red arrow). In terms of LRs, GUS was mainly expressed in mature LRs (Fig. S7C, D) and the bases of immature LRs (Fig. 3C-2, the red arrow). Intriguingly, under the influence of NPA, GUS was only expressed at root tips (Fig. 3D-1, the red and blue arrow). Little signal can be observed in LR bases (Fig. 3D-2, the blue arrow). In sharp contrast, GUS expression was hardly detected in neither mature nor immature roots, with the treatment of AVG (Fig. 3E-1, E-2, the blue arrow). These results suggested that $S p A R F 4$ expression can be induced by auxin.

\section{$S p A R F 4$ influences Cd transportation and accumulation by changing plant architectures}


In order to clarify the functions of SpARF4, it was introduced into S. plumbizincicola and S. alfredii under the control of CaMV 35S promoter, respectively.

In leaf, overexpressing $S p A R F 4$ made leaves distorted and asymmetric, especially in highest-expression lines of $S$. alfredii (Fig. 4A). In addition, the petiole of OE became wider than that of WT (Fig. 4A). Then, these leaves were soaked in ethanol to identify the causes for the phenotypic changes. The results showed that an unambiguous midvein was observed in WT leaves (Fig. 4A, III, the red arrow) and the distribution of lateral veins was stronger symmetry (Fig. 4A, III). However, two midveins can be observed in OE leaves (Fig. 4A, IV, the red arrow and the blue arrow), which may be the reason for a wider petiole. Moreover, the second midvein was wider and run parallel to the first midvein (Fig. 4A, IV). Given that these morphological alterations accorded with typical phenotypes of PIN1 mutation which were conserved in dicots (Mattsson et al., 1999; Sawchuk et al., 2013), we next tested PIN1 expression in WT and OE. The results showed that PIN1 expression was indeed seriously decreased in OE (Fig. 4A, V, P <0.05), indicating that alteration of auxin transportation and distribution may be the reason for leaves phenotypic changes.

In terms of stem, as $A B C G 14$ regulated lignin content and vessel cell numbers while SpARF4 may control SpABCG14 (Fig. 2C), overexpression of SpARF4 could influence the lignification degree and vessel development of transgenic plants. For this reason, stem sections of $\mathrm{OE}$ were made and then stained by phloroglucinol-HCl. At the same time, lignin contents were also tested to verify this assumption. The results indicated that vessel numbers and diameters in $S$. plumbizincicola were all seriously decreased in OE (Fig. 4B, I and II; Fig. S8A, B). Furthermore, there were highly significant decreases in vessel areas and lignin contents (Fig. 4B, III, VI). More than itself of variation in vessels, we focused on whether these changes could modify Cd transportation. The xylem saps of WT and OE were collected at the same time under $\mathrm{Cd}$ stress and testing $\mathrm{Cd}$ content of them. The results showed that no significant difference in $\mathrm{Cd}$ concentration was observed in xylem saps of WT and OE (Fig. S9A). However, the collected saps volume and Cd contents of OE were significantly lower than these of WT (Fig. S9B, P <0.01; Fig. 4B, V, P <0.05). Except for this, we also found that $\mathrm{Cd}$ accumulation in $\mathrm{OE}$ aerial parts were obviously lower than that of WT (Fig. 4B, VI, $\mathrm{P}<0.01$ ).

In root, compared with WT, SpARF4 seriously repressed ARs growth (Fig. 4C, I and II). This inhibition mainly showed in two aspects: one was ARs numbers (Fig. 4C, III) and another was the maximum root length (Fig. 4C, III-2). Given that PIN1 was declined in leaves of OE (Fig. 4A, V), we speculated that the two phenomena may be due to alteration in auxin transportation and distribution of OE. In order to verify this assumption, the auxin reporter gene vector, DR5:GUS, was introduced into WT and OE, respectively. When local auxin accumulation (LAA) and LRs were observed on fibrous roots of WT (Fig. 4C, IV, the white arrow), GUS signals were still uniformly distributed on the same-length fibrous roots of OE and no LR was observed (Fig. 4C, IV-2). Moreover, GUS signals in WT root tips were not only distributed in auxin-produced sites, but also spread to surrounding areas (Fig. 4C, V). Whereas, GUS was only expressed in auxin-produced sites of OE root tips (Fig. 4C, V-2). Taken together, these results strongly indicated that SpARF 4 could delay the rate of LAA to influence root development through disturbing auxin transportation and distribution.

\section{$S p A R F 4$ alters auxin transportation by indirectly regulating $P I N s$}

SpARF4 may affect PIN genes to change auxin transportation and distribution, which was concluded by phenotypic analysis of leaves and roots. To further verify this conclusion, qRT-PCR was made to detect mRNA levels of PINs (PIN1 , PIN2, PIN3 andPIN7) in roots. As we expected, PINs transcripts were seriously repressed in $\mathrm{OE}$ (Fig. $5 \mathrm{~A}, \mathrm{P}<0.01$ ). Intriguingly, promoter analysis showed that no AuxRE element was found in upstream regulatory sequences of PINs (examined by Plantcare,http://bioinformatics.psb.ugent.be/webtools/plantcare/html/; Table 2S). Therefore, we tended to consider that $S p A R F$ 4 indirectly regulate these PINs instead of a direct way.

When measuring ethylene production of WT and OE under Cd stress, we found that ethylene was produced at the beginning of $\mathrm{Cd}$ treatment in both WT and OE (Fig. 5B). After that, ethylene production of WT 
continued to increase, while little increase was found in OE during Cd treatment. Given that ethylene was an important regulator for PIN genes, we cultured WT with supplement of PZA and then tested PINs expression in roots. Interestingly,PINs of WT in this situation were highly similar to these of OE (Fig. 5C). According to these results, we suspected that $S p A R F$ 4indirectly controlled PINs by changing ethylene production. Moreover, $\mathrm{ACO}_{4}$ processing ACC to ethylene was also detected in coexpression network (Hu et al., 2018) (Fig. 2C, the red letter), indicating that ACO4 may be a media between SpARF4 and ethylene.

\section{$S p A R F 4$ negatively controls the transcripts of $\mathrm{SpABCG} 14$ and $\mathrm{SpACO}_{4}$}

According to the above analysis, substantial evidences promoted us to explore whether SpARF4 could

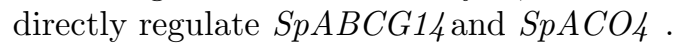

Combining dual-LUC reporter (Fig. 6A), qRT-PCR and Y1H assays, we found that SpARF4 can directly negatively regulate $S p A B C G 14$ and $S p A C O 4$. There were three evidences supporting our conclusion. Firstly, at the protein level, when using Agrobacterium tumefaciens (GV1301) carrying pGreenII 62-SK (EV), pGreenII 62-SK-SpARF4 (SpARF4),SpABCG14pro:LUC and SpACO4pro:LUC to infect Nicotiana benthamiana leaves (Fig. 6B, C), we found that luminescence intensities of 35S:LUC were stronger than these of $S p A B C G 14$ pro:LUC (Fig. 6B, 1 and 2), suggesting that our system was available. Interestingly, when adding $S p A R F 4$, the transient transcriptional activity of $S p A B C G 14$ promoter was significantly repressed (Fig. 6B, 2 and 3). At the mRNA level, adding $S p A R F 4$ made relative mRNA level of Luc lower than that of adding EV (Fig. 6D, P <0.05), suggesting that SpARF4can negatively control SpABCG14. This conclusion was applied equally to the relationship between $S_{p A R F} 4$ and $S_{p A C O}$ (Fig. 6C, E). Secondly, overexpression of $S p A R F 4$ significantly downregulated $S p A B C G 14$ and $S p A C O 4$ in OE, in comparison with these of WT (Fig. 6F and G, P <0.05). Thirdly, Y1H assay indicated that SpARF4 rescued the auxotrophic phenotypes of yeast (Fig. $6 \mathrm{H}$ ), suggesting that SpARF4 can directly bind to AuxRE elements in promoters of $\mathrm{SpABCG14}_{4}$ and $\mathrm{SpACO}_{4}$.

\section{Discussion}

Cd absorption, transportation and sequestration are crucially important for $\mathrm{Cd}$ bioremediation. ABCG14 plays roles in vessel cell development and lignin biosynthesis (Zhao, 2016), which is relevant with Cd transportation and sequestration. In this study, we demonstrated that $S p A R F 4$ was an upstream gene to negatively regulate $S p A B C G 14$ (Fig. $6 \mathrm{~B}$ ). The following evidences are provided to support our conclusions: firstly, $S p A R F$ 4 was prominently expressed in vessel cells of xylem (Fig. 3A); secondly, $S p A R F 4$ can directly bind to the AuxRE motif of $S p A B C G 14$ promoter in $\mathrm{Y} 1 \mathrm{H}$ and negatively regulate $S p A B C G 14$ expression in transient transcription assay (Fig. $6 \mathrm{~B}, \mathrm{H}$ ); thirdly, by contrast with WT, the transcript of SpABCG14 was seriously declined in $\mathrm{OE}$ (Fig. 6F). Besides, genetic phenotypes of OE with declined $S p A B C G 14$ expression exhibited smaller vessel areas (Fig. 4B, II and III) and lower lignin content (Fig. 4B, VI), which were consistent with the ABCG14 mutant phenotypes (Ko et al., 2014; Zhang et al., 2014). In this study, pot experiment was conducted under Cd stress, which may effectively simulate actual scene of bioremediation. The results indicated that $S_{p A R F}$ can negatively regulate $\mathrm{Cd}$ transportation rate of xylem saps and $\mathrm{Cd}$ concentration of $\mathrm{OE}$ aerial tissues (Fig. 4B, V and VI).

Dubrovsky et al. (2008) demonstrated that LAA was a necessary and sufficient signal for LRs formation (Dubrovsky et al., 2008). Another study pointed out that root excision promoted IAA transportation and local accumulation to drive ARs formation (Sukumar et al., 2013). These results emphasize the importance of LAA for root development while genes affecting LAA are related with root development. Here, we demonstrated that $S p A R F 4$ disturbed ethylene biosynthesis by regulating $S_{p A C O}$ (Fig. 5B; Fig. 6C, G, H) and repressed the growth of ARs and LRs (Fig. 4C). Similar to our results, LRs initiation hypothesis points out that an increase of local IAA concentration triggers ethylene production during vessel differentiation of roots, which blocks the normal IAA transportation to form LAA (Aloni et al., 2006). This hypothesis highlights the roles of ethylene in LAA during LRs formation. It has been shown in other studies (Ivanchenko et al., 2008). In a nutshell, SpARF4 is a bridge between auxin and ethylene to function in root development by changing auxin transportation. 
In fact, the crosstalk between auxin and ethylene is not only related with plant's development but also with their adaptation for abiotic stress (Vandenbussche et al., 2010; Su et al., 2015). For example, hormonal network containing ethylene and auxin is crucial for root architecture under osmotic stress (Rowe et al., 2016). Therefore, it is important for us to understand the interactional mechanisms between auxin and ethylene. As early as 2006,ARF7 and ARF19 has been reported as a cross talk between auxin and ethylene in Arabidopsis (Li et al., 2006). Muday et al. (2012) further emphasized the roles of ARFs as a bridge between auxin and ethylene (Muday et al., 2012). But, how ARFs play their roles between auxin and ethylene requires more explanations. Finally, $A R F 7$, as an transcription activator in auxin pathway, is reported to regulate $\mathrm{ACO}_{4}$ to influence ethylene production (Hu et al., 2018). Our study further affirmed that $A R F s$ were involved in ethylene pathway by this mode (Fig. 6C, E, H). Even though, unlike ARFr , $A R F 4$ is a transcription repressor in auxin pathway (Chandler, 2016). Moreover, the coexpression network analysis (containing hub genes, $S p A R F 4,8,19.2$ ) showed that the three genes shared the same related genes (Fig. S6), suggesting that SpARF4, 8, 19.2 were likely to function in the same developmental event. A line of evidences supports this hypothesis. For example,ARF4, 8, 19 in Arabidopsis play their roles in LRs development, respectively (Okushima et al., 2007; Marin et al., 2010; Xi et al., 2018). Thus, it is speculated that $A R F$ family members: $A R F 4,7,8$ and 19 , as important components for auxin signal transduction may function in ethylene pathway. Nonetheless, more studies are necessary to clarify the exact functions of these genes.

According to our results, we uncovered the relationships ofSpARF4, SpABCG14 and SpACO4 . Fig. 7A exhibited the crosstalk between auxin and ethylene. Furthermore, based on these information, a model (Fig. 7B) was proposed to explain the following phenomena: (1) Cd content of aerial tissues was increased under Cd stress (Fig. 4B, VI); (2) More lateral roots were grown when Cd concentration was from 25 to $200 \mu \mathrm{mol} \cdot \mathrm{L}^{-1}$ (Yang et al., 2004); (3) Lignification degree was enhanced during Cd treatment (Fig. 1a); (4) Ethylene was overproduced under Cd stress (Fig. 5B). Based on this model (Fig. 7B), IAA contents are decreased under Cd stress, which leads to a decline in SpARF4 expression. This process enhances the expressions of $S_{p A C O} 4$ and $S p A B C G 14$, as SpARF 4 negatively regulate the two genes. On one hand, more ethylene is produced, which enhances PINs expression. LAA is accelerated and more LRs are grown. More $\mathrm{Cd}$ are thus absorbed by roots. On the other hand, more vessel cells are developed, which can accelerate Cd transportation through apoplastic pathway. Moreover, abundant lignin is biosynthesized to fix $\mathrm{Cd}$ in aerial tissues. And then, Cd accumulation is enhanced through these processes in aboveground tissues.

\section{Acknowledgments}

This work was supported by the Chinese Academy of Forestry foundation (CAFYBB2019QB002), the National Natural Science Foundation of China (No. 31872168); the Zhejiang Science and Technology Major Program on Agricultural New Variety Breeding (No. 2016C020561) and the Agricultural Projects of Public Scientific and Technology Research Zhejiang Province (No. 2016C32G3030016).

\section{Author contributiions}

D.X., X.H., R.Z. designed the research. D.X., X.H. performed the experiments. Z.L., L.W., G.Q., and W.Q. contributed analytical tools, and D.X., X.H. wrote the manuscript and coordinated its revision. R.Z. contributed reagents/materials/funds support. All authors read and provided helpful discussions, and approved the final version.

\section{Declaration of Competing Interest}

None

\section{References}

Ali H, Khan E, Sajad MA (2013) Phytoremediation of heavy metals - Concepts and applications. Chemosphere 91: 869-881

Aloni R, Aloni E, Langhans M, Ullrich C (2006) Role of cytokinin and auxin in shaping root architec- 
ture: regulating vascular differentiation, lateral root initiation, root apical dominance and root gravitropism. Annals of botany 97: 883-893

Benková E, Michniewicz M, Sauer M, Teichmann T, Seifertová D, Jürgens G, Friml J (2003) Local, efflux-dependent auxin gradients as a common module for plant organ formation. Cell 115: 591-602

Blilou I, Xu J, Wildwater M, Willemsen V, Paponov I, Friml J, Heidstra R, Aida M, Palme K, Scheres B (2005) The PIN auxin efflux facilitator network controls growth and patterning in Arabidopsis roots. Nature 433: 39

Casimiro I, Marchant A, Bhalerao RP, Beeckman T, Dhooge S, Swarup R, Graham N, Inzé D, Sandberg G, Casero PJ (2001) Auxin transport promotes Arabidopsis lateral root initiation. The Plant Cell13: 843-852

Chandler JW (2016) Auxin response factors. Plant Cell \& Environment 39: 1014-1028

Daud M, Sun Y, Dawood M, Hayat Y, Variath M, Wu Y-X, Mishkat U, Najeeb U, Zhu S (2009) Cadmium-induced functional and ultrastructural alterations in roots of two transgenic cotton cultivars. Journal of Hazardous Materials 161: 463-473

De Smet I, Lau S, Voß U, Vanneste S, Benjamins R, Rademacher EH, Schlereth A, De Rybel B, Vassileva V, Grunewald W (2010) Bimodular auxin response controls organogenesis in Arabidopsis. Proceedings of the National Academy of Sciences 107: 2705-2710

Demirbas A (2004) Adsorption of lead and cadmium ions in aqueous solutions onto modified lignin from alkali glycerol delignication. Journal of hazardous materials 109: 221-226

Du Y, Scheres B (2017) Lateral root formation and the multiple roles of auxin. Journal of Experimental Botany 69: 155-167

Dubrovsky JG, Sauer M, Napsucialy-Mendivil S, Ivanchenko MG, Friml J, Shishkova S, Celenza J, Benková E (2008) Auxin acts as a local morphogenetic trigger to specify lateral root founder cells. Proceedings of the National Academy of Sciences 105: 8790-8794

Evangelou MWH, Papazoglou EG, Robinson BH, Schulin R (2015) Phytomanagement: Phytoremediation and the Production of Biomass for Economic Revenue on Contaminated Land,

Geisler M, Blakeslee JJ, Bouchard R, Lee OR, Vincenzetti V, Bandyopadhyay A, Titapiwatanakun B, Peer WA, Bailly A, Richards EL(2005) Cellular efflux of auxin catalyzed by the Arabidopsis MDR/PGP transporter AtPGP1. The Plant Journal 44: 179-194

Gu X (2011) Statistical theory and methods for evolutionary genomics,

Gutierrez L, Bussell JD, Păcurar DI, Schwambach J, Păcurar M, Bellini C (2009) Phenotypic plasticity of adventitious rooting in Arabidopsis is controlled by complex regulation of AUXIN RESPONSE FACTOR transcripts and microRNA abundance. The Plant Cell 21:3119-3132

Han X, He G, Zhao S, Guo C, Lu M (2012) Expression analysis of two NAC transcription factors PtNAC068 and PtNAC154 from poplar. Plant molecular biology reporter 30: 370-378

Han X, Yin H, Song X, Zhang Y, Liu M, Sang J, Jiang J, Li J, Zhuo R (2016) Integration of small RNAs, degradome and transcriptome sequencing in hyperaccumulator Sedum alfredii uncovers a complex regulatory network and provides insights into cadmium phytoremediation. Plant Biotechnology Journal 14: $1470-1483$

Harmita H, Karthikeyan K, Pan X (2009) Copper and cadmium sorption onto kraft and organosolv lignins. Bioresource technology100: 6183-6191 
He F, Xu C, Fu X, Shen Y, Guo L, Leng M, Luo K (2018) The microRnA390/TRAnS ACTING SHORT INTERFERING RNA3 module mediates lateral root growth under salt stress via the auxin pathway. Plant Physiology177: pp.01559.02017

He XJ, Mu RL, Cao WH, Zhang ZG, Zhang JS, Chen SY (2005) AtNAC2, a transcription factor downstream of ethylene and auxin signaling pathways, is involved in salt stress response and lateral root development. The Plant Journal 44: 903-916

Hobecker KV, Reynoso MA, Bustos-Sanmamed P, Wen J, Mysore KS, Crespi M, Blanco FA, Zanetti ME (2017) The microRNA390/TAS3 pathway mediates symbiotic nodulation and lateral root growth. Plant physiology174: 2469-2486

Hu J, Israeli A, Ori N, Sun T-p (2018) The Interaction between DELLA and ARF/IAA Mediates Crosstalk between Gibberellin and Auxin Signaling to Control Fruit Initiation in Tomato. The Plant Cell30: $1710-1728$

Iglesias MJ, Terrile MC, Bartoli CG, D'Ippólito S, Casalongué CA (2010) Auxin signaling participates in the adaptative response against oxidative stress and salinity by interacting with redox metabolism in Arabidopsis. Plant molecular biology 74: 215-222

Ivanchenko MG, Muday GK, Dubrovsky JG (2008) Ethylene-auxin interactions regulate lateral root initiation and emergence in Arabidopsis thaliana. The Plant Journal 55: 335-347

Jin X, Yang X, Islam E, Dan L, Mahmood Q (2008) Effects of cadmium on ultrastructure and antioxidative defense system in hyperaccumulator and non-hyperaccumulator ecotypes of Sedum alfredii Hance. Journal of Hazardous Materials 156: 387-397

Ko D, Kang J, Kiba T, Park J, Kojima M, Do J, Kim KY, Kwon M, Endler A, Song W-Y (2014) Arabidopsis ABCG14 is essential for the root-to-shoot translocation of cytokinin. Proceedings of the National Academy of Sciences 111: 7150-7155

Kumar S, Stecher G, Li M, Knyaz C, Tamura K (2018) MEGA X: molecular evolutionary genetics analysis across computing platforms. Molecular biology and evolution 35: 1547-1549

Lee HW, Cho C, Pandey SK, Park Y, Kim M-J, Kim J (2019) LBD16 and LBD18 acting downstream of ARF7 and ARF19 are involved in adventitious root formation in Arabidopsis. BMC plant biology 19: 46

Lewis DR, Negi S, Sukumar P, Muday GK (2011) Ethylene inhibits lateral root development, increases IAA transport and expression of PIN3 and PIN7 auxin efflux carriers. Development 138: 3485-3495

Li J, Dai X, Zhao Y (2006) A role for auxin response factor 19 in auxin and ethylene signaling in Arabidopsis. Plant physiology140: 899-908

Li S-B, Xie Z-Z, Hu C-G, Zhang J-Z (2016) A review of auxin response factors (ARFs) in plants. Frontiers in plant science $\mathbf{7}: 47$

Li T, Yang X, Lu L, Islam E, He Z (2009) Effects of zinc and cadmium interactions on root morphology and metal translocation in a hyperaccumulating species under hydroponic conditions. Journal of hazardous materials 169: 734-741

Liu H, Zhao H, Wu L, Liu A, Zhao FJ, Xu W (2017) Heavy metal ATPase 3 (HMA3) confers cadmium hypertolerance on the cadmium/zinc hyperaccumulator Sedum plumbizincicola. New Phytologist 215:687-698

Liu H, Zhao H, Wu L, Xu W (2017) A genetic transformation method for cadmium hyperaccumulator Sedum plumbizincicola and non-hyperaccumulating ecotype of Sedum alfredii. Frontiers in plant science 8: 1047 
Liu N, Dong L, Deng X, Liu D, Liu Y, Li M, Hu Y, Yan Y (2018) Genome-wide identification, molecular evolution, and expression analysis of auxin response factor (ARF) gene family in Brachypodium distachyon L. BMC Plant Biology 18: 336

Liu Y, Ji X, Nie X, Qu M, Zheng L, Tan Z, Zhao H, Huo L, Liu S, Zhang B (2015) Arabidopsis Atb HLH 112 regulates the expression of genes involved in abiotic stress tolerance by binding to their E-box and GCG-box motifs. New Phytologist 207: 692-709

Liu Y, Wei H, Ma M, Li Q, Kong D, Sun J, Ma X, Wang B, Chen C, Xie Y, Wang H (2019) Arabidopsis FHY3 and FAR1 Regulate the Balance between Growth and Defense Responses under Shade Conditions. The Plant Cell 31: 2089-2106

Lu C, Zhang L, Tang Z, Huang X-Y, Ma JF, Zhao F-J (2019) Producing cadmium-free Indica rice by overexpressing OsHMA3. Environment international 126: 619-626

Lu L-l, Tian S-k, Yang X-e, Wang X-c, Brown P, Li T-q, He Z-l(2008) Enhanced root-to-shoot translocation of cadmium in the hyperaccumulating ecotype of Sedum alfredii. Journal of Experimental Botany 59: 3203-3213

Luo ZB, He J, Polle A, Rennenberg H (2016) Heavy metal accumulation and signal transduction in herbaceous and woody plants: Paving the way for enhancing phytoremediation efficiency. Biotechnology Advances 34: 1131-1148

Marin E, Jouannet V, Herz A, Lokerse AS, Weijers D, Vaucheret H, Nussaume L, Crespi MD, Maizel A (2010) miR390, Arabidopsis TAS3 tasiRNAs, and their AUXIN RESPONSE FACTOR targets define an autoregulatory network quantitatively regulating lateral root growth. The Plant Cell 22: 1104-1117

Mattsson J, Sung ZR, Berleth T (1999) Responses of plant vascular systems to auxin transport inhibition. Development126: 2979-2991

Muday GK, Rahman A, Binder BM (2012) Auxin and ethylene: collaborators or competitors? Trends in plant science 17:181-195

Negi S, Ivanchenko MG, Muday GK (2008) Ethylene regulates lateral root formation and auxin transport in Arabidopsis thaliana. The Plant Journal 55: 175-187

Okushima Y, Fukaki H, Onoda M, Theologis A, Tasaka M (2007) ARF7 and ARF19 regulate lateral root formation via direct activation of LBD/ASL genes in Arabidopsis. The Plant Cell 19: 118-130

Peret B, Swarup K, Ferguson A, Seth M, Yang Y, Dhondt S, James N, Casimiro I, Perry P, Syed A (2012) AUX/LAX genes encode a family of auxin influx transporters that perform distinct functions during Arabidopsis development. The Plant Cell 24: 2874-2885

Paponov IA, Teale WD, Trebar M, Blilou I, Palme K (2005) The PIN auxin efflux facilitators: evolutionary and functional perspectives. Trends in plant science 10: 170-177

Park J, Song WY, Ko D, Eom Y, Hansen TH, Schiller M, Lee TG, Martinoia E, Lee Y (2012) The phytochelatin transporters AtABCC1 and AtABCC2 mediate tolerance to cadmium and mercury. The Plant Journal69: 278-288

Pengcheng F, Jing T, Jingmei H (2008) On the determination of cellulose and lignin of peanut shells. Journal of Chongqing University of Science and Technology: Natural Sciences Edition 10: 64-65

Redjala T, Zelko I, Sterckeman T, Legue V, Lux A (2011) Relationship between root structure and root cadmium uptake in maize. Environmental and Experimental Botany 71: 241-248

Rizwan M, Ali S, Adrees M, Rizvi H, Zia-Ur-Rehman M, Hannan F, Qayyum MF, Hafeez F, Yong SO (2016) Cadmium stress in rice: toxic effects, tolerance mechanisms, and management: a critical 
review. Environmental Science \& Pollution Research 23: 1-21

Rowe JH, Topping JF, Liu J, Lindsey K (2016) Abscisic acid regulates root growth under osmotic stress conditions via an interacting hormonal network with cytokinin, ethylene and auxin. New Phytologist211: 225-239

Sasaki A, Yamaji N, Yokosho K, Ma JF (2012) Nramp5 is a major transporter responsible for manganese and cadmium uptake in rice. The Plant Cell 24: 2155-2167

Sawchuk MG, Edgar A, Scarpella E (2013) Patterning of leaf vein networks by convergent auxin transport pathways. PLoS Genetics9: e1003294

Scanlon MJ (2003) The Polar Auxin Transport Inhibitor $<$ em $>\mathrm{N}</$ em $>$-1-Naphthylphthalamic Acid Disrupts Leaf Initiation, KNOX Protein Regulation, and Formation of Leaf Margins in Maize. Plant Physiology 133: $597-605$

Soeno K, Goda H, Ishii T, Ogura T, Tachikawa T, Sasaki E, Yoshida S, Fujioka S, Asami T, Shimada Y (2010) Auxin biosynthesis inhibitors, identified by a genomics-based approach, provide insights into auxin biosynthesis. Plant and Cell Physiology 51: 524-536

Sorin C, Bussell JD, Camus I, Ljung K, Kowalczyk M, Geiss G, McKhann H, Garcion C, Vaucheret H, Sandberg G (2005) Auxin and light control of adventitious rooting in Arabidopsis require ARGONAUTE1. The Plant Cell 17: 1343-1359

Su L, Diretto G, Purgatto E, Danoun S, Zouine M, Li Z, Roustan J-P, Bouzayen M, Giuliano G, Chervin C (2015) Carotenoid accumulation during tomato fruit ripening is modulated by the auxinethylene balance. BMC plant biology 15: 114

Sukumar P, Maloney GS, Muday GK (2013) Localized induction of the ATP-binding cassette B19 auxin transporter enhances adventitious root formation in Arabidopsis. Plant physiology 162: 1392-1405

Sun X, Li Y, He W, Ji C, Xia P, Wang Y, Du S, Li H, Raikhel N, Xiao J (2017) Pyrazinamide and derivatives block ethylene biosynthesis by inhibiting ACC oxidase. Nature communications 8: 15758

Tao Q, Jupa R, Liu Y, Luo J, Li J, Kovač J, Li B, Li Q, Wu K, Liang Y (2019) Abscisic acid-mediated modifications of radial apoplastic transport pathway play a key role in cadmium uptake in hyperaccumulator Sedum alfredii. Plant, cell \& environment 42:1425-1440

Tao Q, Jupa R, Luo J, Lux A, Kováč J, Wen Y, Zhou Y, Jan J, Liang Y, Li T (2016) The apoplasmic pathway via the root apex and lateral roots contributes to Cd hyperaccumulation in the hyperaccumulator Sedum alfredii. Journal of experimental botany68: 739-751

van de Mortel JE, Schat H, Moerland PD, van Themaat EVL, Van Der Ent S, Blankestijn H, Ghandilyan A, Tsiatsiani S, AARTS MG (2008) Expression differences for genes involved in lignin, glutathione and sulphate metabolism in response to cadmium in Arabidopsis thaliana and the related Zn/Cdhyperaccumulator Thlaspi caerulescens. Plant, Cell \& Environment 31: 301-324

Vandenbussche F, Petrášek J, Žádníková P, Hoyerová K, Pešek B, Raz V, Swarup R, Bennett M, Zažímalová E, Benková E, Van Der Straeten D(2010) The auxin influx carriers AUX1 and LAX3 are involved in auxin-ethylene interactions during apical hook development in <em $>$ Arabidopsis thaliana </em> seedlings. Development137: 597-606

Vidoz ML, Loreti E, Mensuali A, Alpi A, Perata P (2010) Hormonal interplay during adventitious root formation in flooded tomato plants. The Plant Journal 63: 551-562

Wang Y, Li K, Chen L, Zou Y, Liu H, Tian Y, Li D, Wang R, Zhao F, Ferguson BJ (2015) MicroRNA167-directed regulation of the auxin response factors GmARF8a and GmARF8b is required for soybean nodulation and lateral root development. Plant physiology 168: 984-999 
Wilmoth JC, Wang S, Tiwari SB, Joshi AD, Hagen G, Guilfoyle TJ, Alonso JM, Ecker JR, Reed JW (2005) NPH4/ARF7 and ARF19 promote leaf expansion and auxin-induced lateral root formation. The Plant Journal43: 118-130

Xi D, Chen X, Wang Y, Zhong R, He J, Shen J, Ming F (2018) Arabidopsis ANAC092 regulates auxin-mediated root development by binding to the ARF8 and PIN4 promoters. Journal of integrative plant biology

Xie Y, Wang J, Zheng L, Wang Y, Luo L, Ma M, Zhang C, Han Y, Beeckman T, Xu G (2019) Cadmium stress suppresses lateral root formation by interfering with the root clock. Plant, cell \& environment

Xiong L, Wang R-G, Mao G, Koczan JM (2006) Identification of drought tolerance determinants by genetic analysis of root response to drought stress and abscisic acid. Plant physiology 142:1065-1074

Yang X, Long X, Ye H, He Z, Calvert D, Stoffella P (2004) Cadmium tolerance and hyperaccumulation in a new Zn-hyperaccumulating plant species (Sedum alfredii Hance). Plant and Soil 259:181-189

Yang Z (2007) PAML 4: phylogenetic analysis by maximum likelihood. Molecular biology and evolution 24: $1586-1591$

Zhang K, Bhuiya M-W, Pazo JR, Miao Y, Kim H, Ralph J, Liu C-J(2012) An engineered monolignol 4-O-methyltransferase depresses lignin biosynthesis and confers novel metabolic capability in Arabidopsis. The Plant Cell 24: 3135-3152

Zhang K, Novak O, Wei Z, Gou M, Zhang X, Yu Y, Yang H, Cai Y, Strnad M, Liu C-J (2014) ArabidopsisABCG14 protein controls the acropetal translocation of root-synthesized cytokinins. Nature communications 5: 3274

ZHANG ZC, CHEN BX, QIU BS (2010) Phytochelatin synthesis plays a similar role in shoots of the cadmium hyperaccumulator Sedum alfredii as in non-resistant plants. Plant, cell \& environment 33:12481255

Zhao Q (2016) Lignification: flexibility, biosynthesis and regulation. Trends in plant science 21: 713-721

Zhao T, Xu X, Wang M, Li C, Li C, Zhao R, Zhu S, He Q, Chen J(2019) Identification and profiling of upland cotton microRNAs at fiber initiation stage under exogenous IAA application. BMC genomics20: 421

Figure legends

Fig. 1 Variation of lignin and IAA under Cd stress

$\mathbf{a}$ and $\mathbf{b}$ indicated lignin concentration of aerial tissues and $\mathrm{Cd}$ concentration in lignin, respectively, during Cd stress for 0-7 d. c IAA concentration of different organs in WT and OE at the $7 \mathrm{~d}$ under Cd stress. d, day; DW, dry weight; Capital letters indicated $\mathrm{P}$ value $<0.01$ and different letters showed there was a highly significant level between different treatments while the same letter indicated no significant difference was in different treatments. ${ }^{*}, \mathrm{P}<0.05 ; * *, \mathrm{P}<0.01$.

Fig. 2 bioinformatics analysis of $A R F$ gene family

A ARFs number in different species. B Phylogenetic tree of ARF gene families in Kalanchoe laxiflora, Kalanchoe fedtschenkoi and S. plumbizincicola . C coexpression analysis of SpARF4 and the diameters of circles indicated AuxRE number in the promoters of related genes.

Fig. 3 Histological localization of $S p A R F 4$ and strength of GUS signals treated by different inhibitors

A ProSpARF4:GUS signals were detected in xylem and phloem of stem. ph, phloem; xy, xylem; pi, pith. 
B Longitudinal sections showed that a stronger signal of GUS was in vessel elements.

C-1-E-1 The influence of auxin on promoter activities was tested by different inhibitors. C-1 and C-2 without any inhibitors; D-1 and D-2 treated by NPA;E-1 and E-2 treated by AVG. The red arrows pointed GUS signals and the blue arrows indicated that GUS signals in these sites were not detected. NPA, auxin transport inhibitor naphthylphthalamic acid; AVG, auxin synthesis inhibitor aminoethoxyvinylglycine.

Fig. 4 Morphological characters of SpARF 4 overexpression plants

A Developmental malformation of leaves were observed inSpARF4 overexpression lines (OE, II). Compared with WT lines (WT, I), OE leaves were asymmetric and the petioles were wider than that of WT (I and II). Only one midvein was seen in WT (III, the red arrow). By contrast, a second midvein (IV, the blue arrow) was produced and parallel to the first midvein (IV, the red arrow). Moreover, lateral veins of OE were more tanglesome than these of WT (III and IV).PIN1 expression was repressed in OE (V).

B In stem, tissue sections of WT and OE were made and stained by phloroglucinol (I and II). There was a significant difference in vessel areas between WT and OE (III). Meanwhile, lignin contents were seriously decreased in OE (VI). Cd contents in xylem saps and aboveground tissues of OE were significantly declined compared with WT (VI).

C In terms of roots, contrast with WT (I), adventitious roots were repressed in OE (II). The number of adventitious roots were decreased in OE (III) and the maximum root length of OE was also much shorter than that of WT (III-2). When local auxin was accumulated in lateral roots of WT (IV, the white arrow), auxin distribution was uniform in OE lateral roots (IV-2). GUS signal was mainly focused on auxin-production sites in OE root tips (V-2) while the signal was also observed in surrounding area of WT root tips (V).

$*$ indicated $\mathrm{P}<0.05,{ }^{* *}$ indicated $\mathrm{P}<0.01$.

Fig. 5 The effect of $S p A R F 4$ on PINs expression and ethylene production

Overexpression of SpARF 4 can highly significantly repressPINs (PIN1, 2, 3 and 7 ) transcripts (A ). Ethylene in WT and OE was produced at the beginning of $\mathrm{Cd}$ treatment. However, little increase in ethylene production were observed in OE during Cd stress (B ). When culturing WT plants with supplement of pyrazinamide (PZA), expression patterns of the four PINs were similar to these of OE $(\mathbf{C})$. ${ }^{* *}, \mathrm{P}<0.01$.

\section{Fig. $6 S p A R F 4$ directly negatively regulates the expression of $S p A B C G 14$ and $S p A C O 4$}

The full-length sequences of $S p A B C G 14$ and $S p A C O 4$ promoters (2000bp) were constructed into the vector of pGreenII 0800-Luc (A ). Transient transformation assays of $N$. benthamianawere conducted to test the effect of SpARF4 on promoter-driving activities of $S p A B C G 14_{\text {pro }}(\mathbf{B})$ and $S p A C O 4_{\text {pro }}(\mathbf{C})$. The luminescence intensity was shown by the color-scale bar; EV, empty vector pGreenII 62-SK. Relative mRNA levels of $L u c$ were downregulated bySpARF4 ( $\mathbf{D}$ and $\mathbf{E}$ ). Meanwhile, the expression levels of SpABCG14 and SpACO4 in $\mathrm{OE}$ were also seriously repressed in comparison with $\mathrm{WT}(\mathbf{F}$ and $\mathbf{G})$. Y1H assay testing interactions between SpARF 4 and promoter regions ofSpABCG14 and $S_{p A C O}(\mathbf{H})$. The medium containing $30 \mathrm{mM}$ 3 -AT was used for selection, which lacing Trp, Leu and His. Yeast concentration was from 0 to $10^{-3}$. EV1, empty vector pHIS2; EV2, empty vector pGADT7-Rec.

\section{Fig. 7 Model of the crosstalk between auxin and ethylene during Cd stress}

1. Regulatory relationships of components in auxin and ethylene pathway.

2. The roles of SpARF4, SpACO4 and SpABCG14 in Cd absorption, transportation and accumulation of plants under Cd stress. 

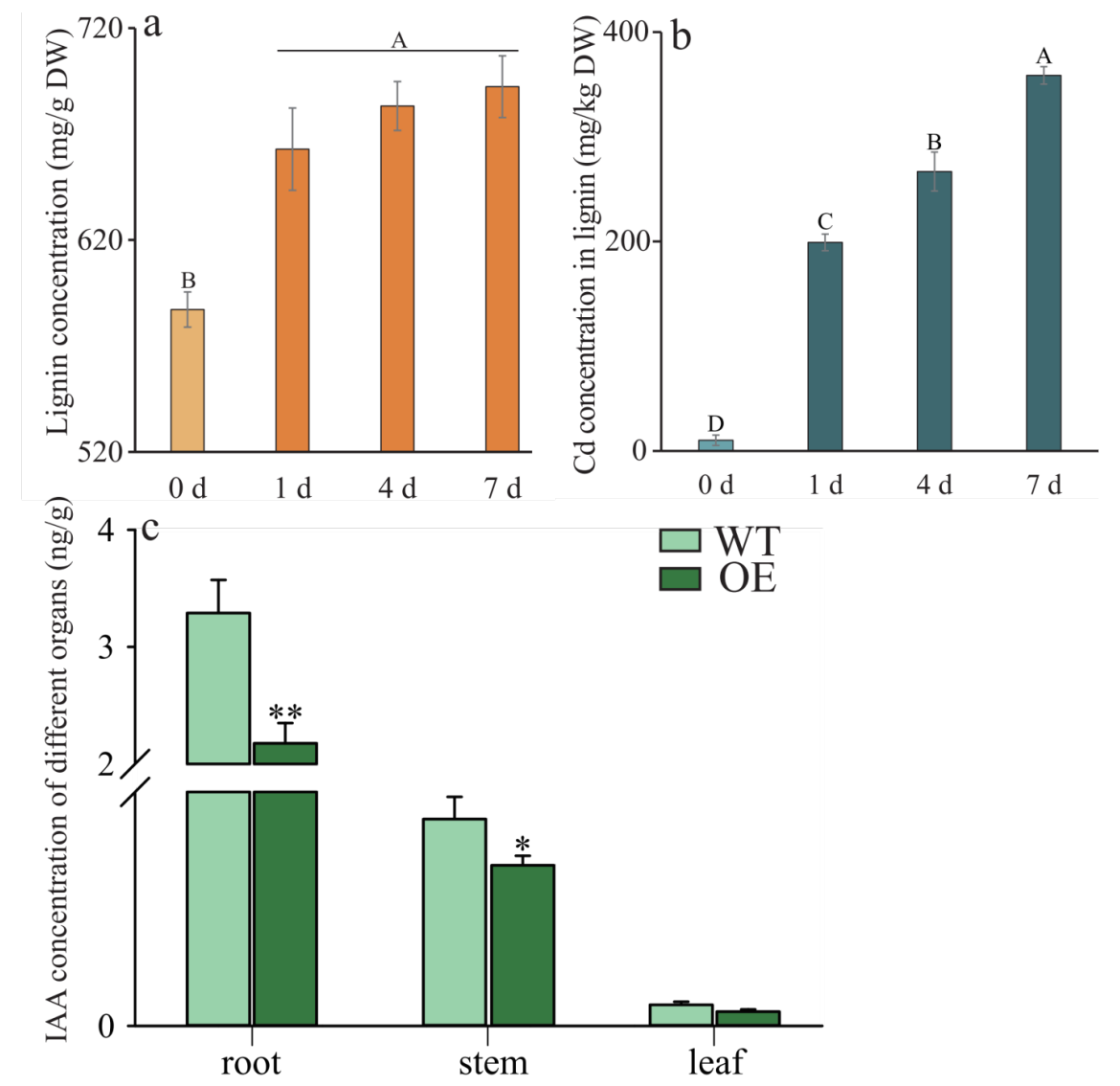

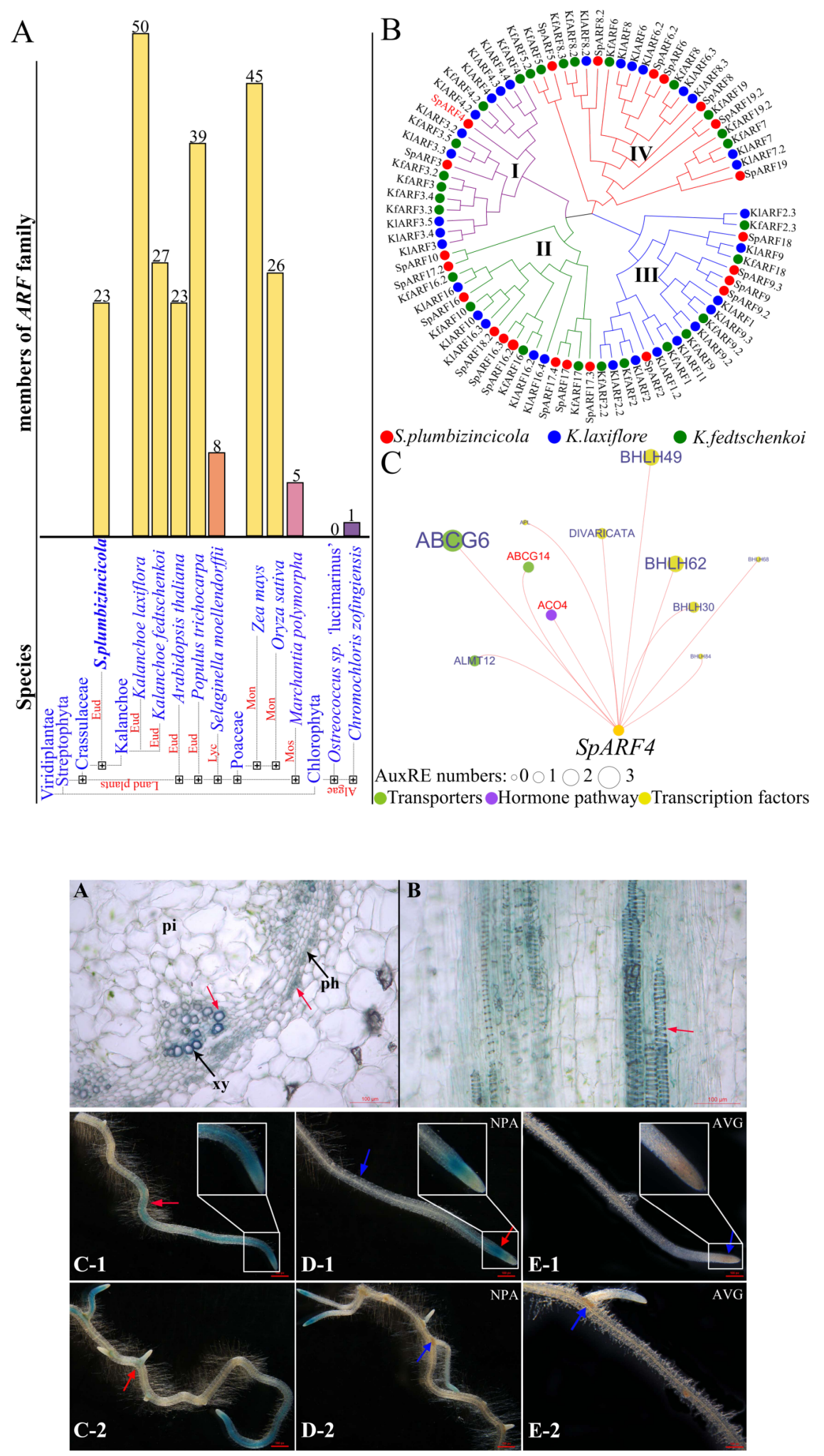

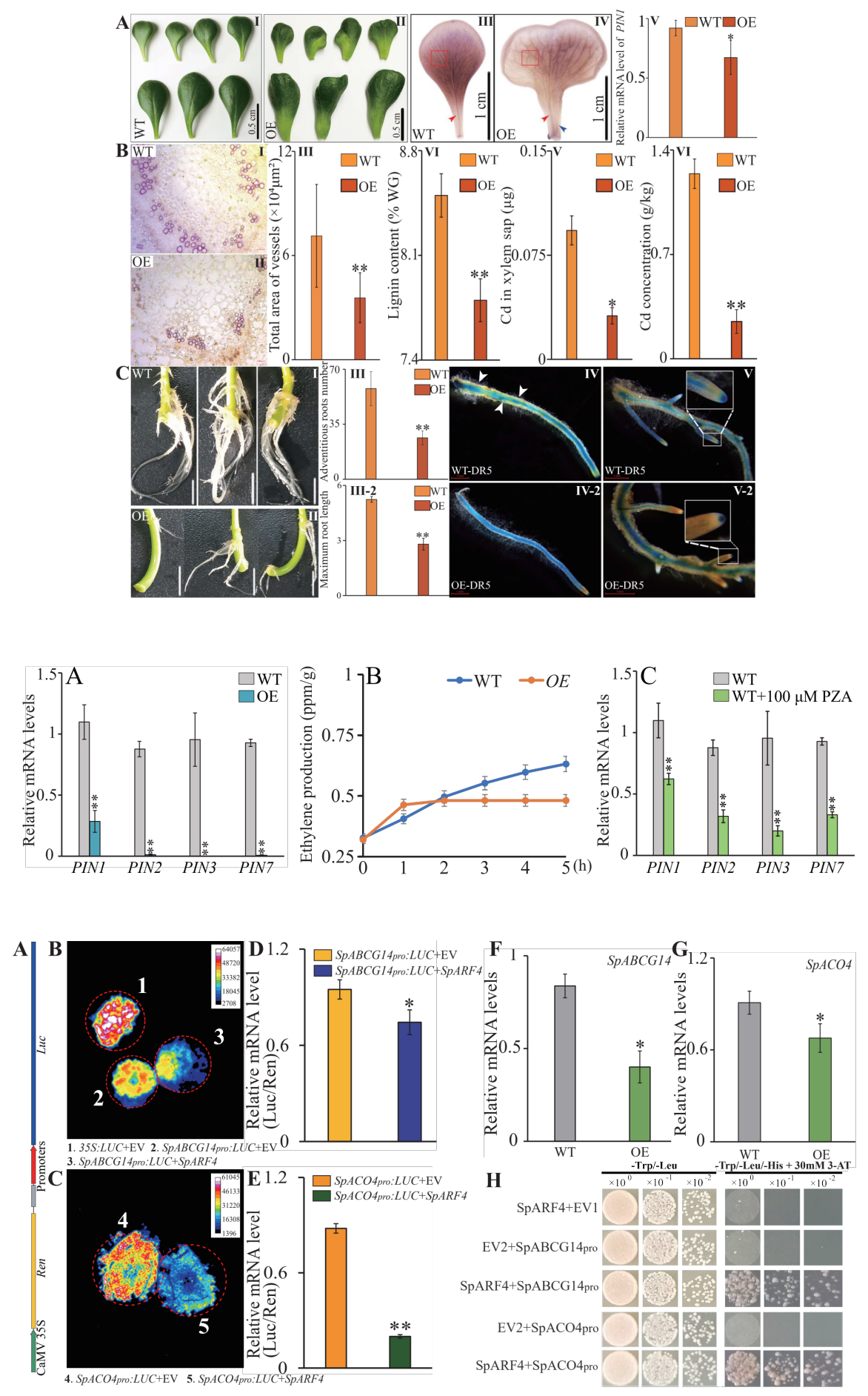


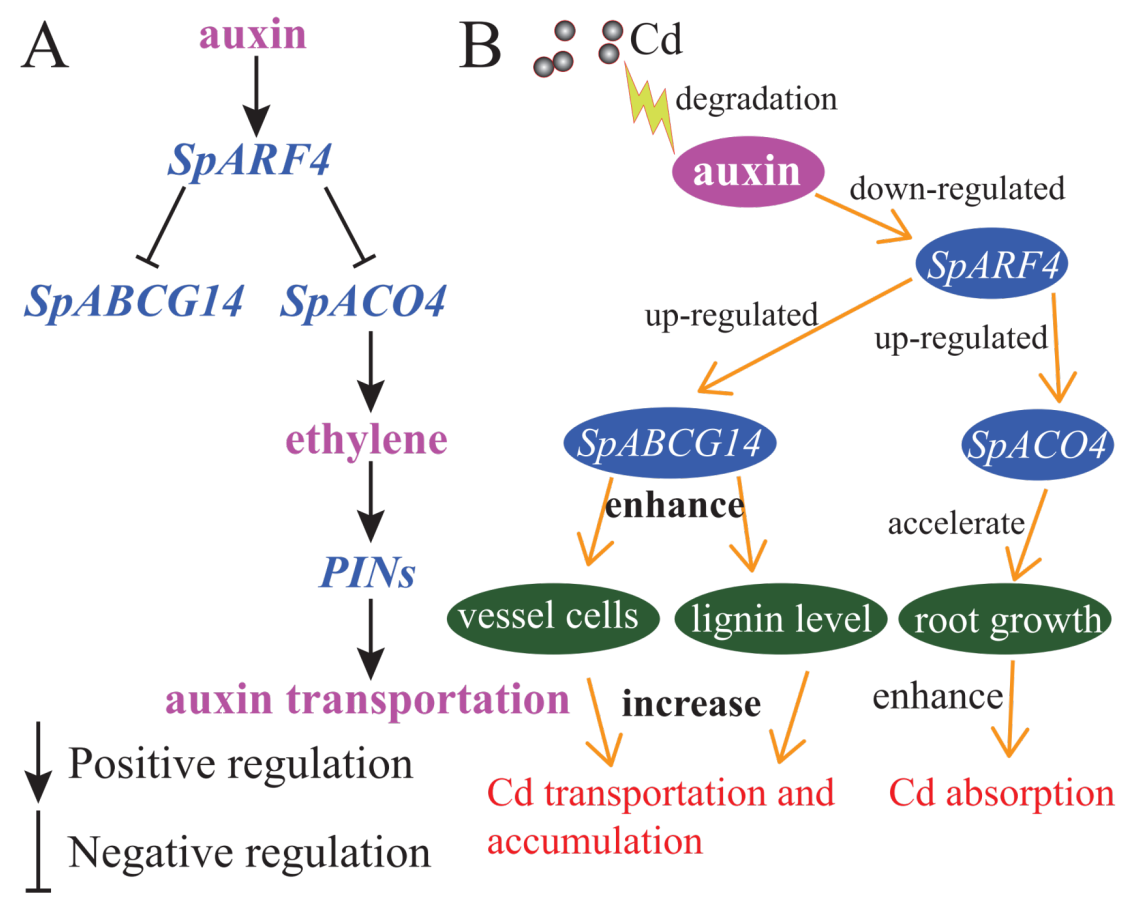

